\title{
Toward explanation of microstructure in pulsar radio emission
}

\author{
S. A. Petrova ${ }^{\star}$ \\ Institute of Radio Astronomy, 4, Chervonopraporna St., Kharkov, 61002 Ukraine \\ Received 7 January 2004 / Accepted 16 February 2004

\begin{abstract}
Propagation of relativistically beamed radio photons through the ultrarelativistic highly magnetized plasma of pulsar magnetosphere is considered. The photons are found to be subject to an additional strong focusing inside the beam because of induced Compton scattering off the plasma particles. Incorporating this focusing effect is shown to improve on the angular hundred times squeezed and its size becomes compatible with the observed micropulse widths. The induced Compton scattering can also account for the polarization properties of micropulses. Furthermore, given a favourable observational geometry, the
\end{abstract} \\ beaming model of pulsar microstructure. For the typical conditions in the scattering region, the photon beam appears up to a few \\ focusing effect can explain the giant micropulses observed in the Vela and some other pulsars.
}

Key words. plasmas - waves - scattering - pulsars: general

\section{Introduction}

Micropulses - intensity fluctuations at microsecond timescales - are known to be a fundamental feature of pulsar radio emission (e.g. Cordes et al. 1990; Hankins 1996; Lange et al. 1998; Popov et al. 2002). For the pulsars studied, they are observed in a large fraction of single pulses over a broad frequency range. The micropulses often appear as an internal structure of subpulses and can show quasiperiodicities within their envelope. The periods of microstructure lie in a broad range, up to a few milliseconds, with the power spectra exhibiting sets of features of different structure and thus proving a general complexity of the micropulse periodicities. As a rule, the lifetime of micropulses is less than the pulsar period and they do not contribute to the average profile. Recent observations of the Vela and some other pulsars (Johnston et al. 2001; Johnston \& Romani 2002; Kramer et al. 2002) have revealed occasional giant micropulses with the intensities up to 100 times the average, which nevertheless are too narrow to enhance the total singlepulse intensities significantly. The giant pulses of the Crab pulsar exhibit a substructure at much shorter timescales, up to a few nanoseconds (Hankins et al. 2003), which is qualitatively similar to the microstructure in normal pulsars and hints at a similar origin.

At present there is no clear understanding of the phenomenon of pulsar microstructure. The micropulses can result from the temporal fluctuations in pulsar plasma, which affect the radio emission process or subsequent radio wave propagation (e.g. Harding \& Tademaru 1981; Chian \& Kennel 1983; Asseo et al. 1990; Gangadhara et al. 1993; Machabeli et al. 2001). Then the associated scale of the plasma inhomogeneities should be $\sim 3 \mathrm{~km}$ (e.g. Popov et al. 2002). Alternatively, the microstructure can be attributed to the non-uniform angular pattern of the emission cone, which manifests itself as the

\footnotetext{
* e-mail: rai@ira.kharkov.ua
}

pulsar rotates (e.g. Cordes 1979; Gil 1982, 1986). This so called beaming model of microstructure is strongly supported by the observations: the characteristic widths of micropulses are proportional to the pulsar period (Hankins 1996; Popov et al. 2002; Kramer et al. 2002), testifying to the same scale of the angular pattern in different pulsars. Because of relativistic beaming, at each point of the emission cone pulsar radiation should be concentrated into a beam of the width $\sim 1 / \gamma$ (where $\gamma$ is the Lorentz-factor of the secondary plasma particles). If one take it as a scale for change in the angular pattern of pulsar emission, then Lorentz-factors as large as $2 \times 10^{4}$ are needed to explain the observed timescales of microstructure (Popov et al. 2002). Such extremely high values of $\gamma$ seem not to be realistic. In other words, the micropulses appear too narrow to be attributed to relativistic beaming.

In the present Letter, we suggest a modified beaming model of microstructure, which allows for propagation effects in pulsar plasma. Namely, the extra-beaming of pulsar radiation is interpreted as a consequence of induced Compton scattering off the plasma particles. According to the previous estimates (e.g. Blandford \& Scharlemann 1976; Lyubarskii \& Petrova 1996), at typical pulsar conditions the optical depth to this process can be large. Then, as the induced scattering is a non-linear process, its consequences can be known only from the exact solution of the corresponding kinetic equation. Below it will be shown that the induced scattering within a narrow beam (with the opening angle $\sim 1 / \gamma$ ) propagating at large enough angle, $\psi$, to the magnetic field, $\psi \gamma \gg 1$, leads to the photon focusing in the direction closest to the magnetic field. This effect is expected to underlie the formation of micropulses of various widths, depending on the physical conditions in the scattering region, such as the plasma number density, beam-field geometry and incident intensity.

The plan of the Letter is as follows. In Sect. 2 we solve the kinetic equation for the photons undergoing the induced 
scattering off the particles of the ultrarelativistic highly magnetized plasma. The solution obtained is applied to pulsar case in Sect. 3. In Sect. 4 we discuss our results in the context of the observed properties of microstructure. Section 5 contains a brief summary.

\section{Induced scattering of pulsar radiation}

Let a beam of radio photons propagate through the ultrarelativistic highly magnetized plasma of pulsar magnetosphere. As the brightness temperatures of pulsar radio emission are extremely high, $T \sim 10^{25}-10^{30} \mathrm{~K}$, the photons can undergo the induced scattering off the plasma particles. Given that the photon frequency in the plasma rest frame is much less than the electron gyrofrequency, $\omega \prime \ll \frac{e B}{m c}$, the magnetic field affects the scattering process, changing both the particle recoil and the scattering cross-section. As is shown in Lyubarskii \& Petrova (1996), at pulsar conditions the induced scattering in the limit of a strong magnetic field always dominates the scattering in the weak field and can be pronounced at distances of order of the emission altitude, where the plasma density is large enough. In the laboratory frame, the evolution of the photon occupation numbers, $n(\boldsymbol{k})$, is described by the following kinetic equation (e.g. Blandford \& Scharlemann 1976, Eq. (30)):

$$
\begin{aligned}
& \frac{\partial n(k, \theta, \phi)}{\partial t}+c \frac{\partial n(k, \theta, \phi)}{\partial r}=\frac{\hbar}{m} n_{\mathrm{e}} r_{\mathrm{e}}^{2} n(k, \theta, \phi) \\
& \times \int \frac{\sin ^{2} \theta \sin ^{2} \theta_{1}}{\gamma^{6} \eta^{3} \eta_{1}^{3} \beta^{2}}\left\{\frac{1}{\gamma^{3}}\left(\frac{\eta_{1}-\eta}{\eta_{1}}\right)^{2} \frac{\partial}{\partial k}\left[k_{1}^{2} n_{1}\left(k_{1}, \theta_{1}, \phi_{1}\right)\right]\right. \\
& \left.+\frac{6 k \eta^{2}\left(\eta_{1}-\eta\right) n_{1}\left(k_{1}, \theta_{1}, \phi_{1}\right)}{\gamma \eta_{1}^{2}}\left[1-\frac{1}{2 \gamma^{2}}\left(\frac{\eta_{1}+\eta}{\eta \eta_{1}}\right)\right]\right\} \theta_{1} \mathrm{~d} \theta_{1} \mathrm{~d} \phi_{1}
\end{aligned}
$$

where $(k, \theta, \phi)$ are the spherical coordinates of the wavevector $\boldsymbol{k}$ in the system with the polar axis along the axis of the photon beam, $n_{1}\left(k_{1}, \theta_{1}, \phi_{1}\right)$ is the occupation number of the photons involved in the scattering process, and the integral is taken over the beam; $r_{\mathrm{e}}$ is the classical electron radius, $n_{\mathrm{e}}$ the particle number density, $\beta$ the particle velocity in units of $c, \eta \equiv 1-\beta \cos \vartheta_{b}$, with $\vartheta_{b}$ being the angle between the wavevector and the particle velocity, and

$k \eta=k_{1} \eta_{1}$.

The latter equality implies that in the proper frame of the scattering particles the incident and scattered photons have the same frequency. Below we consider the stationary case and the term $\frac{\partial n}{\partial t}$ will be omitted. Further simplification is possible, if one consider a narrow beam, $\theta \lesssim 1 / \gamma$, which propagates at an angle $\psi \gg 1 / \gamma$ to the magnetic field. Then the first term on the right-hand side of Eq. $(1)$ is $(\psi \gamma)^{3}$ less than the second one and can be neglected. Moreover, in this case $\eta \approx \eta_{1}$ for any photon direction inside the beam, so that the photons are scattered only between the states with very close frequencies. To the eldest order in $(\psi \gamma)^{-1}$, Eq. (1) is reduced to the following form:

$$
\begin{aligned}
\frac{\partial n(k, \theta, \phi)}{\partial a r}= & n(k, \theta, \phi) \int_{0}^{2 \pi} \int_{0}^{1 / \gamma} n_{1}\left(k, \theta_{1}, \phi_{1}\right) \\
& \times\left[\theta \cos \phi-\theta_{1} \cos \phi_{1}\right] \theta_{1} \mathrm{~d} \theta_{1} \mathrm{~d} \phi_{1}
\end{aligned}
$$

where

$a=384 \hbar k n_{\mathrm{e}} r_{\mathrm{e}}^{2} /(m c)(\psi \gamma)^{-7}$

and the azimuth $\phi=0$ corresponds to the plane containing the beam axis and magnetic field direction.

It is easy to notice that the kinetic Eq. (3) has the first integral:

$N=\int n(k, \theta, \phi) \theta \mathrm{d} \theta \mathrm{d} \phi=$ const.

i.e. in the assumed approximation the number of photons of a given frequency is conserved and the scattering acts only to redistribute the photon directions within the beam. The kinetic Eq. (3) describes the evolution of the photon occupation number, $n(k, \theta, \phi)$, in an arbitrary state inside the beam. From the two similar equations for the two arbitrary states, $n_{a}\left(k, \theta_{a}, \phi_{a}\right)$ and $n_{b}\left(k, \theta_{b}, \phi_{b}\right)$, one can find:

$\frac{\partial\left(n_{a} / n_{b}\right)}{\partial a r}=\frac{n_{a}}{n_{b}} N\left(\theta_{a} \cos \phi_{a}-\theta_{b} \cos \phi_{b}\right)$.

Hence,

$\frac{n_{a}}{n_{a_{0}}}=\frac{n_{b}}{n_{b_{0}}} \frac{\exp \left(N a r \theta_{a} \cos \phi_{a}\right)}{\exp \left(N a r \theta_{b} \cos \phi_{b}\right)}$.

Taking $n_{a_{0}} \approx n_{b_{0}}$ and using Eq. (5), we integrate both sides of Eq. (7) over the solid angle $\theta_{a} \mathrm{~d} \theta_{a} \mathrm{~d} \phi_{a}$ to obtain the occupation number $n_{b}$ in an arbitrary state inside the beam:

$n_{b}=\frac{N \exp \left(N a r \theta_{b} \cos \theta_{b}\right)}{2 \pi / \gamma^{2}(N a r / \gamma)^{-1} I_{1}(N a r / \gamma)}$

Here $I_{1}(N a r / \gamma)$ is the modified Bessel function of the first order. Equation (8) can be rewritten in terms of the spectral intensity of the beam, using a well-known relation, $i_{v}=2 h v^{3} n / c^{2}$ :

$i_{\nu}(\theta, \phi)=\frac{I_{\nu} \exp \left(I_{\nu} \widetilde{a} r \theta \cos \phi\right)}{2 \pi / \gamma^{2}\left(I_{\nu} \widetilde{a} r / \gamma\right)^{-1} I_{1}\left(I_{\nu} \widetilde{a} r / \gamma\right)}$,

with

$\widetilde{a}=a c^{2} /\left(2 h v^{3}\right)$.

As is evident from Eq. (9), the evolution of the spectral intensity $i_{v}(\theta, \phi)$ in the course of induced scattering is determined by the factor $\Gamma \equiv I_{\nu} \widetilde{a} r / \gamma$. Given that $\Gamma \ll 1 I_{1}(\Gamma) \approx \Gamma / 2$ and we come to the initial uniform intensity distribution: $i_{v}(\theta, \phi)=$ $I_{\nu}\left(\pi / \gamma^{2}\right)^{-1}$. In the opposite limit, $\Gamma \gg 1, I_{1} \approx \exp (\Gamma) / \sqrt{2 \pi \Gamma}$ and

$i_{\nu}(\theta, \phi) \approx \frac{I_{\nu}}{\pi / \gamma^{2}} \sqrt{\frac{\pi}{2}} \Gamma^{3 / 2} \exp [\Gamma(\gamma \theta \cos \phi-1)]$.

One can see that the radiation tends to be focused toward $\theta_{f}=$ $1 / \gamma, \phi_{f}=0$, which defines the direction closest to the magnetic field. For a given $\Gamma$, the radiation is concentrated within the region $\Delta \theta \sim(\Gamma \gamma)^{-1}, \Delta \phi \sim 1 / \sqrt{\Gamma}$, whereas $i_{v}\left(\theta_{f}, \phi_{f}\right) \propto \Gamma^{3 / 2}$.

In the above consideration, we have ignored the difference in the frequencies of the photons participating in the scattering. More exactly, Eq. (5) implies the conservation of the number of photons obeying Eq. (2). Substituting $\eta \approx \psi^{2}(1-2 \theta \cos \phi / \psi) / 2$ 
in Eq. (2), one can find that in reality the scattering process involves the photons over the frequency range $\Delta v / v \sim$ $(\psi \gamma)^{-1}$. Further on, as the photons are focused in the region $\Delta(\theta \cos \phi) \sim(\Gamma \gamma)^{-1}$, their frequencies are concentrated in the interval $\Delta v / v \sim(\Gamma \psi \gamma)^{-1}$ near the highest one. Thus, the focusing effect is accompanied by an increase of the photon energy, but the energy gain is insignificant because of the beam narrowness.

The photons satisfying the condition $k \eta=$ const. participate in the induced scattering independently of the others. Correspondingly, for the broad-band beam the results are qualitatively the same. It should be mentioned, however, that $\Gamma$ is a strong function of frequency, $\Gamma \propto v^{-2} I_{v}$, implying the higher efficiency of the induced scattering at the lower frequencies. Let us also note in passing that for the widely spaced frequencies the angle $\psi$ can be different (e.g. because of difference in the emission altitudes or because of differential action of propagation effects in pulsar plasma), and therefore the frequency dependence of $\Gamma$ can differ essentially from that given above.

\section{Numerical estimates}

Now let us estimate the efficiency of induced Compton scattering for the conditions relevant to pulsar magnetospheres. Substituting Eqs. (4) and (10), we have

$\Gamma=\frac{192 n_{\mathrm{e}} r_{\mathrm{e}}^{2} I_{v} r(\psi \gamma)^{-7}}{m \gamma v^{2}}$.

The spectral intensity can be presented as $I_{v}=I_{v_{0}}\left(v / v_{0}\right)^{-\alpha}$, with

$I_{v_{0}}=\frac{L}{v_{0} \pi r^{2} w^{2} / 4}$

and $v_{0} \sim 10^{8} \mathrm{~Hz}$. Here $L$ is the total pulsar luminosity, $w$ the pulse width in the angular measure, $w \equiv 2 \pi \tau / P$, with $\tau$ being the pulse width in the temporal measure, $P$ the pulsar period. It is convenient to introduce the multiplicity factor of the secondary plasma, $\kappa$, which is the ratio of the particle number density to the Goldreich-Julian density: $\kappa=N P c e / B$. Then we obtain:

$$
\begin{aligned}
\Gamma= & 340 P \frac{L}{10^{28} \mathrm{erg} \mathrm{s}^{-1}} \frac{B_{\star}}{10^{12} \mathrm{G}} \frac{\kappa}{10^{2}}\left(\frac{\tau}{1 \mathrm{~ms}}\right)^{-2}\left(\frac{\gamma}{10^{2}}\right)^{-1} \\
& \times\left(\frac{v}{10^{8} \mathrm{~Hz}}\right)^{-\alpha-2}\left(\frac{r}{10^{8} \mathrm{~cm}}\right)^{-4}\left(\frac{\psi}{10^{-1}} \frac{\gamma}{10^{2}}\right)^{-7},
\end{aligned}
$$

where $B_{\star}$ is the magnetic field strength at the surface of the neutron star and the stellar radius is taken to be $10^{6} \mathrm{~cm}$. As is evident from Eq. (14), induced scattering can play a substantial role. Of course, $\Gamma$ is the largest for the pulsars with the largest luminosities, magnetic field strengths and plasma multiplicities. It should be noted that $\Gamma$ depends drastically on the location of the scattering region and the scattering geometry. Although in the modern theories of pair creation cascade $\kappa \sim 10^{2}$ is an upper limit for the plasma multiplicity (e.g. Hibschman \& Arons 2001; Arendt \& Eilek 2002) and the microstructure is observable up to the frequencies of a few gigahertz (Lange et al. 1998), it is still possible that the induced Compton scattering is efficient enough to account for the necessary focusing.
As is found in the previous section, given that $\Gamma \gg 1$, the photon beam appears $\Gamma$ times squeezed, i.e.

$w_{\mu} \sim(\Gamma \gamma)^{-1}$ and $\tau_{\mu} \sim 10^{-3} P \Gamma \gamma / 100$

The observations yield the mean width of the short-scale micropulses $w_{\mu} \sim\left(2 \times 10^{4}\right)^{-1}$ (Popov et al. 2002); for the typical Lorentz-factors of the plasma particles, $\gamma \sim 100$, this implies $\Gamma \sim 200$. According to our estimate (14), such high values of $\Gamma$ can indeed be met in pulsars.

Within the framework of our interpretation, the microstructure is associated with the resolvable angular structure of a photon beam, which is set up by the efficient induced scattering off the plasma particles. Then the maximum width of the micropulses should be $w_{\mu_{\max }} \sim 1 / \gamma$, in which case $\Gamma \lesssim 1$. The observations also testify to the existence of the maximum timescale of microstructure: $\tau_{\mu_{\max }} \sim 200 \mu$ s for PSR B0950+08 and B1929+10 and $\tau_{\mu_{\max }} \sim 800 \mu \mathrm{s}$ for PSR B1133+16 (Popov et al. 2002). Substituting the pulsar periods $(P=0.253 \mathrm{~s}$, $0.226 \mathrm{~s}$ and $1.188 \mathrm{~s}$, respectively) into Eq. (15) and taking $\Gamma=1$ and $\gamma=100$ yield $\tau_{\mu_{\max }} \sim 200 \mu$ s for the former two pulsars and $\tau_{\mu_{\max }} \sim 1 \mathrm{~ms}$ for the latter one. These values are in a good agreement with the observed ones. Thus, the characteristic timescales of microstructure can be explained in terms of the beam focusing as a result of induced scattering. Note that the diversity of $\tau_{\mu}$ met in a given pulsar may hint at substantial variations of the physical conditions in the scattering region.

It is interesting to inspect whether the focusing effect can also account for the energetics of the giant micropulses observed at the periphery of the profile of the Vela pulsar in addition to the ordinary microstructure seen throughout the pulse. The narrowness of the giant micropulses as compared to the normal ones hints at the stronger focusing effect resulting from the distinct scattering geometry. The giant micropulses have been detected at a resolution of $44 \mu$ s (Johnston et al. 2001), which corresponds to the resolution of the beam angular pattern $\sim(10 \gamma)^{-1}$ for this pulsar $(P \approx 0.09 \mathrm{~s}$ and $\gamma \sim 100)$. Then the minimum resolvable area of the emission cone is $\sim 100$ times less than the initial cross-section of the photon beam. Provided that the emission of the beam is strongly focused within the resolvable area in the direction detectable by an observer, the intensity appears $\sim 100$ times increased. Hence, the giant micropulses can in principle be attributed to the strong focusing effect in conjunction with a particularly favourable observational geometry. However, more observations are needed to decide as to whether giant micropulses are indeed a sort of microstructure or whether they are linked to giant pulses, where true energy gain is inevitable.

Note that the nanostructure of the giant pulses can also be explained in terms of the focusing effect, so that the problem of exceedingly high brightness temperatures of the nanopulses (Hankins et al. 2003) is removed naturally. A detailed study of the origin of giant pulses and their nanostructure is a subject of a forthcoming paper (Petrova 2004).

\section{Discussion}

The induced scattering of radio photons inside a narrow beam propagating through the ultrarelativistic highly magnetized 
pulsar plasma appears to be significant. We have found that the photons tend to be focused in the direction closest to the ambient magnetic field. The focusing effect is believed to give rise to pulsar microstructure.

Based on Eq. (14) one cannot conclude about the broadband frequency behaviour of the scattering efficiency, since the location of the scattering region and the scattering geometry are expected to be in some way frequency-dependent. Although the microstructure is generally a broad-band phenomenon, in some cases it is correlated only over a small range (e.g. $\Delta v / v \lessgtr 0.1$, Popov et al. 1987). Within the framework of our model, this small scale of frequency correlation can be interpreted as the frequency interval over which the photons of a given frequency can be scattered. As is found at the end of Sect. 2, $\Delta v / v \sim$ $(\Gamma \psi \gamma)^{-1}$; then the observed scale of frequency correlation can be obtained taking $\psi \gamma \sim 10$ and $\Gamma \gtrsim 1$.

It should be noted that the problem of induced scattering considered above concerns only the ordinary waves, which are polarized in the plane containing the wavevector and the ambient magnetic field. For the extraordinary waves (with the electric vector perpendicular to that plane) the scattering crosssection is negligible. Pulsar radiation is generally a mixture of both types of the natural waves, so that only a part of radiation effectively participates in the induced scattering and the consequent focusing. Thus, the micropulses should predominantly consist of the focused ordinary waves and have peculiar polarization properties. This is indeed proved by the observations (e.g. Cordes \& Hankins 1977). Firstly, the linear polarization of micropulses is particularly strong. Secondly, at the edge of a micropulse the position angle frequently jumps by $90^{\circ}$, indicating that out of the focusing direction the intensity of the ordinary waves is dominated by that of the extraordinary ones. Recent studies of the Vela pulsar (Kramer et al. 2002) have revealed the lack of orthogonal transitions in the linear polarization of the micropulses, confirming the dominance of a single polarization mode. The position angle swing is not smooth, showing "wiggles" with the micropulse timescales, whereas the circular polarization is strong, occasionally of the opposite sense. These feature of the microstructure are likely the consequence of a marked polarization evolution in the plasma of pulsar magnetosphere.

The numerical estimates of the previous section yield the microstructure timescales consistent with the observed ones. Note that this estimates are based on the assumption that a micropulse is emitted by a point source, i.e. it is associated with a single photon beam. In another words, it is taken that the beams are focused toward an observer only within separate small regions in the cross-section of the open field line tube. This is valid, if the beam-field geometry, which determines the direction of focusing, changes rapidly across the tube. In the present Letter, we leave aside the problem as to how the focused photon beams from all over the tube form the total angular distribution of intensity observed as microstructure. Below we present only some general comments on the matter. A jitter of the beamfield orientation can be attributed to the presence of the currents which act to disturb the dipolar structure of the magnetic field at each point of the tube. Hence, the pattern of microstructure, including its quasiperiodicities, is likely set up by the global electrodynamics of pulsar magnetosphere, in which aspect it is relative to subpulses. Besides that, the beam-field geometry can be affected by refraction of rays in the inhomogeneous plasma of pulsars.

\section{Conclusions}

We have considered the induced Compton scattering of radio photons off the particles of the ultrarelativistic highly magnetized plasma of pulsar magnetosphere. It is found that owing to this process the relativistically beamed radiation appears substantially redistributed within the beam. If the beam axis is inclined at a large angle to the ambient magnetic field, $\psi \gg 1 / \gamma$, the photons tend to be focused toward the magnetic field direction. The numerical estimates show that at pulsar conditions the induced scattering can be efficient and the photon beam can be squeezed up to a few hundred times. Such scales of the beam pattern are in a good accordance with the timescales of micropulses. Thus, the radio photon beam with the angular structure modified by the focusing effect can be recognized as an elementary constituent of pulsar microstructure. Within the framework of this interpretation, the polarization properties of micropulses are explained naturally. In addition, the focusing effect can account for the giant micropulses, if only a favourable observational geometry is postulated.

Acknowledgements. I am grateful to the referee for the useful comments and suggestions. This research is in part supported by INTAS Grant (call 2003).

\section{References}

Arendt, P. N. Jr., \& Eilek, J. A. 2002, ApJ, 581, 451

Asseo, E., Pelletier, G., \& Sol, H. 1990, MNRAS, 247, 529

Blandford, R. D., \& Scharlemann, E. T. 1976, MNRAS, 174, 59

Chian, A. C. L., \& Kennel, C. F. 1983, Ap\&SS, 97, 9

Cordes, J. M. 1979, Aust. J. Phys., 32, 9

Cordes, J. M., \& Hankins, T. H. 1977, ApJ, 218, 484

Cordes, J. M., Weisberg, J. M., \& Hankins, T. H. 1990, AJ, 100, 1882

Gangadhara, R. T., Krishan, V., \& Shukla, P. K. 1993, MNRAS, 262, 151

Gil, J. 1982, A\&A, 115, 270

Gil, J. 1986, A\&A, 308, 691

Hankins, T. H. 1996, in Pulsars: Problems \& Progress, ed. S. Johnston, M. A. Walker, \& M. Bailes, ASP Conf. Ser., 105, 197

Hankins, T. H., Kern, J. S., Weatherall, J. C., \& Eilek, J. A. 2003, Nature, 422, 141

Harding, A. K., \& Tademaru, E. 1981, ApJ, 243, 597

Hibschman, J. A., \& Arons, J. 2001, ApJ, 554, 624

Johnston, S., van Straten, W., Kramer, M., \& Bailes, M. 2001, ApJ, 549, L101

Johnston, S., \& Romani, R. 2002, MNRAS, 332, 109

Kramer, M., Johnston, S., \& van Straten, W. 2002, MNRAS, 334, 523

Lange, C., Kramer, M., Wielebinski, R., \& Jessner, A. 1998, A\&A, 332,111

Lyubarskii, Yu. E., \& Petrova, S. A. 1996, Astron. Lett., 22, 399

Machabeli, G., Khechinashvili, D., Melikidze, G., \& Shapakidze, D. 2001, MNRAS, 327, 984

Petrova, S. A. 2004, in preparation

Popov, M. V., Smirnova, T. V., \& Soglasnov, V. A. 1987, SvA, 31, 529

Popov, M. V., Bartel, N., Cannon, W. H., et al. 2002, A\&A, 396, 171 